\section{Commentary: A novel cross-species model of Barlow's disease to biomechanically analyze repair techniques in an ex vivo left heart simulator}

\author{
Norman Briffa, MB, MD, FRCS(CTh), FFPC(Ed)
}

The eponymously named "Barlow's disease" of the mitral valve was first described by John Barlow in patients with a late systolic murmur, ${ }^{1}$ a systolic click and aneurysmal change of the posterior leaflet. The changes in Barlow disease of the mitral valve are characterized by thickened enlarged multisegmented leaflets, whose main body prolapses into the left atrium during systole. Histologically there is myxoid degeneration, collagen fragmentation, and accumulation of glycosaminoglycans ${ }^{2}$ within the morphologically abnormal leaflets. Phenotypic changes of the different components of the mitral valve complex have been described in detail, and proteomic studies of diseased leaflets have identified molecules involved in the degenerative process. ${ }^{3}$ Genome-wide association studies have also defined the heritability that is known to occur in these patients. $^{4}$

Barlow's disease is thought to occupy part of the degenerative mitral valve prolapse spectrum, which also includes fibroelastic deficiency ${ }^{2}$ and whose prevalence in the general population is believed to be approximately $2 \%$ to $5 \% .^{5}$

Patients with Barlow's disease of the mitral valve come to our attention when they develop significant mitral valve regurgitation. The mechanism of mitral valve reflux in Barlow's disease can be complex and includes chordal elongation with or without rupture resulting in segmental prolapse.

\footnotetext{
From the Sheffield Teaching Hospitals NHS Trust, University of Sheffield, Chesterman Wing, Northern General Hospital, Sheffield, United Kingdom.

Disclosures: Author has nothing to disclose with regard to commercial support.

Received for publication Jan 23, 2020; accepted for publication Jan 25, 2020; available ahead of print Feb 8, 2020.

Address for reprints: Norman Briffa, MB, MD, FRCS(CTh), FFPC(Ed), Sheffield Teaching Hospitals NHS Trust, Chesterman Wing, Northern General Hospital, Herries Rd, Sheffield S5 7AU, United Kingdom (E-mail: n.briffa@sheffield.ac. uk).

J Thorac Cardiovasc Surg 2021;161:1784-5 0022-5223/\$36.00

Crown Copyright $\odot 2020$ Published by Elsevier Inc. on behalf of The American Association for Thoracic Surgery

https://doi.org/10.1016/j.jtcvs.2020.01.055
}

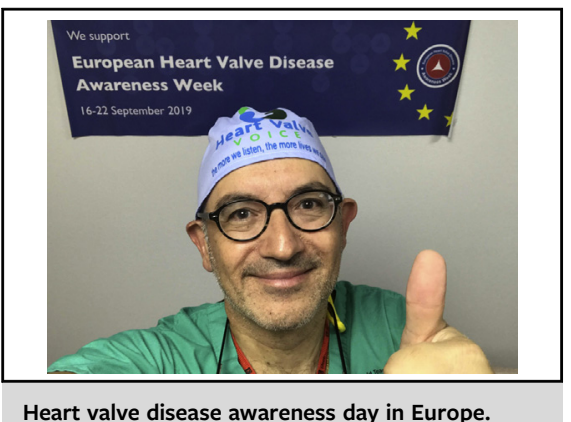

Heart valve disease awareness day in Europe.

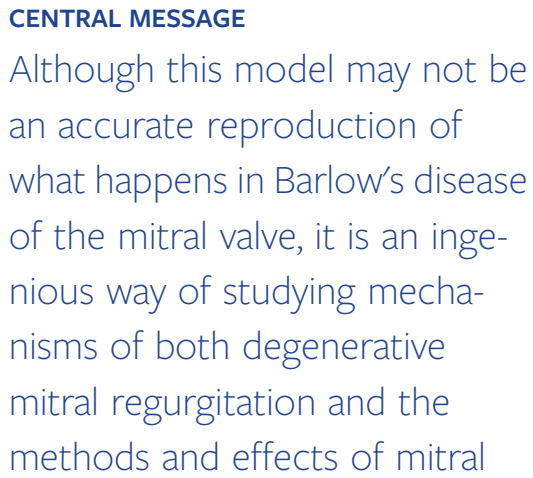

valve repair.

In the absence of chordal rupture, mitral regurgitation in classic Barlow's disease is caused by a dilated annulus, flattened leaflets with loss of coaptation, and systolic abnormalities of the lateral wall of the left ventricle and accompanying papillary muscles. ${ }^{6,7}$ This abnormal movement results in the papillary muscles to displace toward the left atrium and in the absence of coaptation, in regurgitation through the whole line of closure.

As with the rest of the mitral valve prolapse spectrum, durable mitral valve repair is the preferred method of surgical correction. Associated with the multiple mechanisms causing regurgitation, many repair techniques have been described to treat mitral regurgitation in these patients using both classic sternotomy or minimally invasive approaches.

In this article, Woo and colleagues ${ }^{8}$ at Stanford describe the development of what they call a cross-species model to study the biomechanics of Barlow's disease of the mitral valve. They have used a bovine mitral valve in a relatively undersized porcine annulus and variable vertically placed papillary muscles. The segmentation cause by symmetric undersizing results in leaflets that look Barlowesque in appearance.

This whole arrangement has been implanted inside a locally designed previously described circuit that simulates the left heart. This circuit includes a receiving chamber, the 
cross-species mitral model, and a pumping chamber with a mechanical pump emptying into a resistance circuit through a valve (aortic). The pulsatile ( 80 beats $/ \mathrm{min}$ ) pump is designed to produce a blood pressure of $120 / 80$ and a flow of $5 \mathrm{~L}$ per minute. Flow and pressure are accurately measured in the different parts of the circuit. Tiny sensors are placed on the main 4 chordae to continuously measure strain. The proportional undersizing of the bovine valve with elevation of the neopapillary muscles produces Barlow's-like segmented leaflets that leak. Repair of the valve by implantation of neochordae drastically reduces the regurgitant volume and reverses the changes seen in blood pressure, flow, and chordal strain.

This ingenious and meticulously assembled model is the first to produce a model of mitral regurgitation that has not involved transection of chords or artificial stretching of the mitral annulus but has created (relatively) abnormal leaflets. Regurgitation is induced by undersizing of annulus relative to the size of mitral leaflet together with a relative radical shortening of chordae. Undersizing is achieved by using a porcine annulus to support bovine leaflets. Such relative undersizing can equally be produced by the use of a suitable designed and sized artificial round annulus. To use a porcine annulus in this cross-species model seems to be an unnecessary complication.

As I have described earlier, in the absence of isolated chordal elongation or rupture, the driver of mitral regurgitation in Barlow's disease is annular dilation with loss of coaptation. This concept is proven by the fact that the sole implantation of a relatively undersized annular ring/ band, the sizing of which is based on intetrigonal distance, often achieves mitral competence. ${ }^{9}$ In vivo, additional maneuvers are required to avoid systolic anterior motion. The mitral regurgitation created in this model by the undersizing of the annulus does not fully reflect the mechanism in real life.

Designing a model to reflect a specific disease process in a meaningful way is always difficult. Barlow's disease is complex. ${ }^{10}$ It is a heritable genetic disease with complex biological processes affecting the whole mitral valve complex resulting in 3-dimensional physical changes that prevent the valve from achieving hemodynamic competence. Trying to create a model that reflects what happens hemodynamically/biomechanically, let alone one that reproduces biological and genetic abnormalities, is difficult. Nonetheless, the Stanford team have managed to create a unique model that can be used to understand various mechanisms that can cause mitral regurgitation and that can be used to study the effect of the different types of mitral valve repair. The model can also be used as a valuable teaching tool for surgeons in training to ensure high standards for surgeons who want to specialize in surgery of the mitral valve.

\section{References}

1. Barlow JB, Bosman CK. Aneurysmal protrusion of the posterior leaflet of the mitral valve. An auscultatory-electrocardiographic syndrome. Am Heart J. 1966;71:166-78

2. Hjortnaes J, Keegan J, Bruneval P, Schwartz E, Schoen FJ, Carpentier A, et al Comparative histopathological analysis of mitral valves in Barlow disease and fibroelastic deficiency. Semin Thorac Cardiovasc Surg. 2016;28:757-67.

3. Markby GR, Summers KM, MacRae VE, Corcoran BM. Comparative transcriptomic profiling and gene expression for myxomatous mitral valve disease in the dog and human. Vet Sci. 2017;4(3).

4. Gasser S, Reichenspurner H, Girdauskas E. Genomic analysis in patients with myxomatous mitral valve prolapse: current state of knowledge. BMC CardiovasC Disord. 2018:18:41.

5. Althunayyan A, Petersen SE, Lloyd G, Bhattacharyya S. Mitral valve prolapse. Expert Rev Cardiovasc Ther. 2019;17:43-51

6. Lawrie GM. Barlow disease: simple and complex. J Thorac Cardiovasc Surg. 2015;150:1078-81.

7. Ehlers KH, Engle MA, Levin AR, Grossman H, Fleming RJ. Left ventricular abnormality with late mitral insufficiency and abnormal electrocardiogram. Am J Cardiol. 1970;26:333-40.

8. Imbrie-Moore A, M PMJ, Yuanjia Z, Wang Hanjay LHJ, Farry JM, MacArthur JW, et al. A novel cross-species model of Barlow's disease to biomechanically analyze repair techniques in an ex vivo left heart simulator. J Thorac Cardiovasc Surg. 2021;161:1776-83.

9. Lawrie GM, Earle EA, Earle NR. Nonresectional repair of the Barlow mitral valve: importance of dynamic annular evaluation. Ann Thorac Surg. 2009;88: 1191-6.

10. Okechukwu IB. Introductory chapter. In: Bartholomew I, ed. Experimental Animal Models of Human Diseases. London, UK: IntechOpen; 2018. 